\title{
6th International Meeting on Progress in Radio-Oncology, ICRO/ÖGRO 6
}

\author{
May 13-17, 1998, Salzburg, Austria
}

The 6th International Meeting on Progress in Radio-Oncology, ICRO/ÖGRO 6, was organized by the Austrian International Club for Radio-Oncologists (ICRO) of the Austrian Society for Radiooncology, Radiobiology and Medical Radiophysics (ÖGRO). The conference took place from May 13 to 17, 1998, at the Salzburg Residenz, and 14 major topics related to progress in radiooncology were covered. Leading scientists of the various fields from more than 30 countries of all five continents attended this congress. The 877-page proceedings of the meeting include 107 scientific contributions [including two papers by P. Rubin (Rochester) and L.W. Brady (Philadelphia) on angioplastic irradiation] [1].

In the first introductory paper by H.D. Suit (Boston), the problem of local regrowth of a tumor as a source of metastases was discussed. According to Suit, there is solid evidence that distant metastases do develop from the recurring neoplasm. This does mean that there will be some increment in diseasefree survival with each decrement in frequency of distant metastasis. For every gain in local control, there will be improvements in disease-free survival and quality of life. (Currently, $90-95 \%$ of all cancer cures are still entirely due to local treatment with radiotherapy and surgery.)

The second introductory paper by L. Milas et al. (Houston) covered the implications of growth factors and cytokines for radiotherapy. It is the opinion of the authors that intervention at various steps in growth factor signaling pathways opens new avenues for cancer treatment by radiotherapy.

W.H. McBride et al. (Los Angeles) gave the state-of-the-art lecture on new molecular and genetic approaches aimed at improving radiation therapy; strategies combining radiation therapy with certain gene therapy approaches, such as p53 gene replacement, are reasonable and worthy of further exploration.

The field of boron neutron capture therapy (BNCT) was discussed by several speakers from Germany. Clinical trials of BNCT with epithermal neutrons have started for glioblastoma in the United States and in Europe, and the question arises whether and how BNCT can be applied to other tumor entities.

Radiosurgery and stereotactic (fractionated) radiotherapy have gained an outstanding importance in the treatment of both benign and malignant primary intracranial neoplasms.
J. Loeffler (Boston) and R.-P. Müller (Köln) were the main lecturers in this field. There was a rapid development of technical modifications, especially the introduction of linear acceleratorbased radiosurgery and stereotactic radiotherapy for clinical use. Besides several established clinical indications for intracranial neoplasmas, the scope of diseases amenable to this treatment is expanding quickly.

The therapeutic gain by hyperthemia added to radiotherapy was presented by J. van der Zee (Rotterdam) on behalf of the International Collaborative Hyperthermia Group. As an example, a recently closed study in patients with inoperable intrapelvic tumors has demonstrated an improvement in CR rate from 39 to $55 \%$. In patients with cancer of the uterine cervix, the addition of hyperthermia to standard radiotherapy also improved overall survival: from 24 to $48 \%$ after 3 years of follow-up.

Critical issues in mechanisms of photodynamic therapy (PTD) were reported by B.W. Henderson from the Roswell Park Cancer Institute in Buffalo. While PDT is obtaining regulatory approvals for treatment of a wide range of malignant and some nonmalignant conditions, research continues to elucidate the mechanisms responsible for its clinical effectiveness. The cellular and tissue targets of PDT are numerous, resulting in a very complex interaction of many diverse effects.

A new model for the dissemination of regional and distant metastases with implications for the sequencing of treatment of early breast cancer was introduced by H.D. Thames (Houston). Based on fits to data describing the incidence of metastases as a function of volume of primary breast cancer at the time of surgery, the predictions of the modelling are: the rate of successful seeding per cubic centimeter of the primary decreases rapidly as the primary grows, and the estimated seeding frequency is sufficiently high that neoadjuvant treatment of early breast cancer should be reconsidered.

For the treatment of subclinical metastases, H.R. Withers (Los Angeles) suggested two implications for adjuvant therapy:

1) There should be no delay in its initiation because those micrometastatic deposits that are most easily curable will 'escape' the benefit of even relatively ineffective chemotherapy or low doses of $\mathrm{x}$-rays.

$\begin{array}{ll}\text { KARGER } & \text { (1) 1998 S. Karger GmbH, Freiburg } \\ & \text { Fax (0761) 4520714 } \\ \text { www.karger.com } & \text { Accessible online at: } \\ \text { http://BioMedNet.com/karger }\end{array}$


2) Even relatively low doses of $x$-irradiation, if given promptly, may significantly reduce the incidence of metastases. This distinguishes the treatment of subclinical metastases from that of primary tumors, for which low doses are completely ineffective in achieving cure.

The key presentations in the session radiosensitizers/radioprotectors were delivered by G.E. Adams and D.J. Chaplin, and by S. Dische and M.I. Saunders, all from the Mount Vernon Hospital in Northwood. While the former research group discussed new approaches for the exploitation of hypoxia for tumor therapy, Dische and Saunders talked about the clinical relevance of hypoxic cell radiosensitization in 1998. A major effort has now been made to identify those tumors where hypoxia is a major problem leading to radioresistance, and where real benefit might be gained. The tests employed have yielded a great variety of results, but this must be expected. It seems unlikely that any single one or even a combination will become a feature of routine clinical practice. The accumulation of biological knowledge however, correlated with the clinical and histological features, gives promise that in the future we will be able to individualize patient's treatment. This will only be advanced by intensified international cooperation. One major issue of the congress addressed the optimal combination of chemotherapy and radiotherapy, and K.K. Ang (Houston) gave the main lecture. The primary object for combining chemotherapy with radiation treatment should guide the design of multi-modality therapy regimens to maximize the likelihood of yielding therapeutic benefit. Chemotherapy aiming for reduction of the distant metastatic relapse rate, for example, is best comprised of agents proven to have high antitumor activity and tolerance in the treatment of patients with metastatic disease; it is also logical to administer chemotherapy sequentially with radiation (i.e., in an adjuvant or neoadjuvant setting) to prevent occurrence of toxicity resulting from drug-radiation interaction. For improving the probability of local-nodal disease control, however, it is perhaps more rational to choose cytotoxic agents based on their mechanisms of action to counteract known causes of radioresistance rather than based on their independent antitumor acitivity and to time chemotherapy administration such as to yield maximal drug interaction or to prevent specific normal tissue toxicity.

In the session on intraoperative radiotherapy (IORT), experts of leading cancer centers from all over the world [e.g., F. A. Calvo (Madrid), J.P. Gérard (Lyon), M. Abe (Kyoto), L. Gunderson (Rochester), H. Frommhold (Freiburg)] presented their results. When data from international IORT expert institutions using a similar methodology are analyzed site by site, they are quite comparable, which emphasizes the concept that reproducibility of IORT results is high. Intraoperative electron integration in complex treatment sequences for the multidisciplinary management of cancer patients as a treatment component is a solid reality. Further investigations and new technological developments will firmly introduce this treatment modality into the cancer medicine of the next century.

Predictive assays for characterization of individual tumor biology were discussed in a specific session by an international expert panel [L. Peters (Melbourne), J.F. Fowler (London/ Leuven), S. Bodis (Zürich), A.C. Begg (Amsterdam), C. Streffer
(Essen), Y. Shibamoto (Kyoto), C.M.L. West (Manchester), M. Molls (München), P. A. Coucke (Lausanne)]. The definition of prognostic factors is essential to the practice of oncology. Tumor parameters such as tissue of origin, size, extent of local, regional and distant spread, histologic grade, biochemical and molecular markers as well as patient characteristics such as performance status provide powerful prognostic information but do not shed any light on the underlying mechanisms involved. For optimal therapeutic intervention, the cure-limiting attribute of each tumor needs to be known so that a causespecific approach could be taken to therapy.

Conformal radiotherapy (volume effect and 3-D planning) is another issue of major interest relating to progress in radiooncology. Also this topic was covered by an international expert group, with the key lectures given by M. Goitein (Boston), W. Schlegel (Heidelberg), and K.R. Trott (London). Goitein presented the potential benefits and costs of achieving a high degree of conformity of external beam radiotherapy, and Schlegel discussed the new technological developments in conformal radiotherapy. In the paper by Trott about the state of our biological understanding of dose-volume effects it was concluded that we need prospective clinical experiments that are specifically designed to identify the critical structures or elements for each critical organ or tissue, their anatomical position and their radiation response. For the first time, due to the development of high precision 3-D planning, we have now the possibility to do this sort of research.

In the session on particle beam irradiation, representatives from the world's leading centers [e.g., A. Wambersie (Brussels), G. Kraft (Darmstadt), J. Debus (Heidelberg), G. Goitein (Villigen), H. Tsujii (Chiba), H.D. Suit (Boston), J.M. Slater and C. Ross (Loma Linda)] reported about the latest advances of radiotherapy with protons, neutrons and heavy ions. These radiation qualities were introduced in cancer therapy either to improve the radiobiological differential effect, or to improve the physical selectivity of the irradiation, or both (e.g., heavy ions). Heavy ion beam radiotherapy is now developing in several countries because of the ultimate dose distribution that can be monitored by PET and the increased relative biological effectiveness in the tumor. Clinical results with protons and heavy ions (such as carbon ions) seem to be very promising in certain tumors. However, results of randomized trials are still to be seen.

In the conference session on altered fractionation (hyperfractionation, accelerated fractionation), results from major research centers were presented, especially in the field of head and neck cancer [e.g., J.C. Horiot (Dijon), J. Overgaard (Aarhus), M.I. Saunders (Northwood) and B. Maciejewski (Gliwice)]. Horiot presented the results of two randomized EORTC trials, which constitute a milestone in the understanding of dose, fractionation and overall treatment-time relationships in head and neck cancers:

1) Hyperfractionation using 2 fractions per day allows the delivery of $80 \mathrm{~Gy}$ with better locoregional control than with 70 Gy using a single fraction per day, without increasing the incidence and severity of normal tissue damage.

2) Accelerated fractionation, despite a higher acute and late toxicity, led to a significant increase of locoregional control, progression-free survival and overall survival. 
J. Bourhis from Villejuif (on behalf of the MACH-NC Collaborative Group) discussed the meta-analysis on the role of chemotherapy in combination with radiation therapy in head and neck cancer. A total of 76 trials with 11,659 patients were identified, but only 63 trials were available for this analysis. According to the chemotherapy timing, no benefit was gained with adjuvant or neoadjuvant chemotherapy. However, evidence indicates that concomitant or alternating radio-chemotherapy conferred a benefit, but the results in this group are too heterogeneous to allow definitive conclusions. A standard arm without chemotherapy is therefore still needed for future trials.

Finally, one session of the meeting was devoted to cancer cure with organ preservation, which is possible with the help of radiotherapy in up to $40 \%$ of curable patients.

H.D. Kogelnik, F. Sedlmayer, Salzburg

\section{Reference}

1 Kogelnik HD, Sedlmayer F: Progress in Radio-Oncology VI. Bologna, Monduzzi, 1998.

6th International Meeting on Progress in

Radio-Oncology, ICRO/ÖGRO 